\title{
Synchronization of vectorial noise-sustained structures
}

\author{
Gonzalo Izús \\ Departamento de Física, FCEyN, Universidad Nacional de Mar del Plata and CONICET, Funes 3350, (7600) Mar del Plata, Argentina \\ Pere Colet and Maxi San Miguel \\ Instituto Mediterráneo de Estudios Avanzados, IMEDEA (CSIC-UIB), E-07071 Palma de Mallorca, Spain \\ Marco Santagiustina \\ Istituto Nazionale di Fisica della Materia, Dipartimento di Elettronica ed Informatica, Università di Padova, via Gradenigo 6/a, \\ 35131 Padova, Italy \\ (Received 25 April 2003; published 3 September 2003)
}

\begin{abstract}
The synchronization of vectorial, noise-sustained structures in nonlinear optical systems is discussed. In particular, the analysis is made for nondegenerate optical parametric oscillators with walk off. The interplay between walk off and noise fluctuations leads to the formation of noise-sustained transverse patterns in both the signal and idler fields. Despite the fact that both patterns are stochastic macroscopic structures driven by independent sources of noise, their correlation grows with time, finally leading to a spatially distributed time synchronization of noise-sustained structures. A physical explanation of this phenomenon is found by analyzing the linear instability process and the existence of exact nonlinear solutions that show the same correlation.
\end{abstract}

DOI: 10.1103/PhysRevE.68.036201

PACS number(s): 05.45.Xt, 42.65.Sf, 42.65.Yj

\section{INTRODUCTION}

Synchronization phenomena have been a topic of scientific research for many years [1] and in many systems, ranging from physics to biology [2]. Many different situations have been considered, including synchronization of limit cycle oscillators [3,4], synchronization of chaotic systems [5], partial (i.e., phase) synchronization [6], generalized synchronization [7,8], synchronization of stochastic systems [9], noise-induced synchronization [10], etc. These works refer to systems characterized by a purely temporal dynamics. Synchronization phenomena have also been studied in systems with spatial degrees of freedom, in particular, synchronization of two spatiotemporally chaotic fields [11,12]. In Refs. $[11,12]$ the two chaotic fields are taken as the two independent components of a vector field, as for example, the two polarization components of an electric field vector. Synchronization is mediated by the dynamics of spatially localized vectorial structures. In this paper we address the question of the dynamical synchronization of two spatiotemporal fields that follow stochastic dynamics driven by independent noise sources. Again we take these two fields as two independent components of a vector field. To deal with a specific example, we consider noise-sustained patterns of the vectorial electric field, which occur in a model for type-II optical parametric oscillators (OPO's) [13].

Pattern formation has been studied in a variety of nonlinear optical systems [14], being OPO's a particularly interesting example. There are a number of theoretical and experimental studies of pattern formation, localized structures, and domain walls in these systems [15-29]. The effect of the spatial walk off, due to the crystal birefringence, has also been addressed in OPO's. Walk off breaks the reflection symmetry and leads to convective terms in the dynamical equations. Therefore, a convectively unstable regime [30-32] exists. This regime is characterized by the fact that local perturbations of the steady state are advected more rapidly than their rate of spreading. Therefore, macroscopic patterns named noise-sustained structures (NSS's) emerge in this regime if noise is present at all times. Optical noise-sustained structures [33] have been predicted in OPO's [13,30,34]. Such NSS's are conceptually equivalent to those observed in fluid convection experiments, in an open flow configuration [35]. In fluid dynamics the NSS are a spatial macroscopic manifestation of amplified thermal fluctuations, while in OPO's these macroscopic structures are associated with amplified quantum noise. We will consider here type-II OPO in which the nonlinear crystal is pumped by a field linearly polarized with frequency $\omega_{0}$ and the two fields produced with frequencies $\omega_{1,2}$ (signal and idler) are orthogonally polarized. A NSS appears in each of these two components of the vector electric field [13] and we investigate the synchronization of these two stochastic fields.

The paper is organized as follows. Section II briefly reviews the dynamical equations describing transverse patterns in type-II OPO as well as the determination of the threshold for the convective and absolute instabilities. In addition we give numerical evidence of the synchronization phenomena between the two NSS in the convective regime. Section III gives a first justification of the synchronization phenomena in terms of a linear analysis that identifies directions of instability, while Sec. IV gives a further justification of the synchronization in terms of nonlinear solutions of the problem. Section V gives a quantitative characterization of the degree of synchronization. Our conclusions are summarized in Sec. VI.

\section{MEAN-FIELD EQUATIONS, THRESHOLD ANALYSIS, AND SYNCHRONIZATION}

\section{A. Equations for a type-II OPO}

The equations describing the time evolution of the normalized slowly varying envelopes of the transverse electric 
fields in a frequency and/or polarization nondegenerate OPO (NDOPO) have been derived in Refs. [13,25,26,31]. The equations governing the interaction of $A_{0,1,2}(\vec{r}, t)$, i.e., the pump, signal, and idler fields, at frequencies $\omega_{0,1,2}$ (where $\left.\omega_{0}=\omega_{1}+\omega_{2}\right)$, in a NDOPO in a ring cavity, in the meanfield approximation, are given by

$$
\begin{aligned}
\partial_{t} A_{0}= & \gamma_{0}\left[-\left(1+i \Delta_{0}\right) A_{0}+E_{0}+i a_{0} \nabla^{2} A_{0}+2 i K_{0} A_{1} A_{2}\right] \\
+ & \sqrt{\epsilon_{0}} \xi_{0}(\vec{r}, t) \\
\partial_{t} A_{1}= & \gamma_{1}\left[-\left(1+i \Delta_{1}\right) A_{1}+i a_{1} \nabla^{2} A_{1}+i K_{0} A_{2}^{*} A_{0}\right] \\
& +\sqrt{\epsilon_{1}} \xi_{1}(\vec{r}, t), \\
\partial_{t} A_{2}= & \gamma_{2}\left[-\left(1+i \Delta_{2}\right) A_{2}+i a_{2} \nabla^{2} A_{2}+i K_{0} A_{1}^{*} A_{0}+\rho \partial_{y} A_{2}\right] \\
+ & \sqrt{\epsilon_{2}} \xi_{2}(\vec{r}, t),
\end{aligned}
$$

where the paraxial and the single longitudinal mode approximations are assumed to be valid for all the fields. Without loosing generality the fields $A_{0,1}$ can be taken as ordinary polarized beams, while $A_{2}$ is extraordinary polarized. The vector $\vec{r}=(x, y)$ is the spatial coordinate, transverse to the direction of propagation, $t$ is the time, and $K_{0}$ is the nonlinear coefficient. The transverse Laplacian operator describes diffraction, $a_{j}(j=0,1,2)$ being the diffraction coefficients. Due to the birefringence of the nonlinear crystal, $a_{1}$ and $a_{2}$ can be slightly different, even when the signal and idler are frequency degenerate. $E_{0}$ is the normalized amplitude of the injected pump, which is considered real with no loss of generality; $\gamma_{j}$ and $\Delta_{j}(j=0,1,2)$ are, respectively, the cavity decay rates and detunings; and $\rho$ is the walk-off coefficient. By proper scaling of space, time, and amplitudes all these coefficients can be taken as dimensionless; the relations of these parameters with physical quantities can be found in Refs. [15,30,31]. Finally, the last terms of each equation are independent complex Gaussian white noises, with zero mean value and correlation

$$
\left\langle\xi_{i}(\vec{r}, t) \xi_{j}^{*}\left(\vec{r}^{\prime}, t^{\prime}\right)\right\rangle=2 \delta_{i, j} \delta\left(\vec{r}-\vec{r}^{\prime}\right) \delta\left(t-t^{\prime}\right) .
$$

\section{B. Threshold analysis}

Equations (1)-(3) have a homogeneous symmetric solution: $A_{1}=A_{2}=0, A_{0}=E_{0} /\left(1+i \Delta_{0}\right)$, which corresponds to the regime of no-signal generation. The linearization of Eqs. (1)-(3) around this solution identifies eigenvalues with dispersion relations [13]

$$
\begin{aligned}
\lambda_{ \pm}(\vec{q}, F)= & -\frac{1}{2}\left\{\left(\gamma_{1}+\gamma_{2}\right)+i\left[\left(\gamma_{1} \Delta_{1}-\gamma_{2} \Delta_{2}\right)\right.\right. \\
& \left.\left.+q^{2}\left(\gamma_{1} a_{1}-\gamma_{2} a_{2}\right)-\gamma_{2} \rho q_{y}\right]\right\} \pm \frac{1}{2} \sqrt{\chi},
\end{aligned}
$$

where new parameters have been defined in order to simplify the notation, i.e., $\tilde{a}=\gamma_{1} a_{1}+\gamma_{2} a_{2}, \tilde{\Delta}=\gamma_{1} \Delta_{1}+\gamma_{2} \Delta_{2}, \quad \chi$ $=4 \gamma_{1} \gamma_{2}|F|^{2}-\left[q^{2} \tilde{a}+\tilde{\Delta}+\gamma_{2} \rho q_{y}-i\left(\gamma_{1}-\gamma_{2}\right)\right]^{2}$. Finally, $F$ $=i K_{0} E_{0} /\left(1+i \Delta_{0}\right)$ is a normalized pump intensity.

By increasing the pump amplitude $E_{0}$ (i.e., $|F|$ ) the homogeneous symmetric solution becomes unstable. As dis- cussed in Refs. [13,30,31], due to the convective term (proportional to $\rho$ ), this system has actually two thresholds of instability in the parameter $|F|$. The lowest one $\left(F_{c}\right)$ corresponds to the convective instability and it is the threshold of the signal generation for the OPO. Unstable modes are transverse traveling waves (TW's) $A_{1}, A_{2}^{*} \simeq \exp \left[i \vec{q}_{c} \cdot \vec{r}+\lambda_{+}\left(\vec{q}_{c}\right) t\right]$ whose critical wave vector $\left(\vec{q}_{c}\right)$ and frequency $\left(\operatorname{Im}\left[\lambda_{+}\left(\vec{q}_{c}\right)\right]\right)$ are given below. Defining $q_{0, y}=-\gamma_{2} \rho / 2 \tilde{a}$, the convective threshold is $F_{c}=1$ if $q_{0, y}^{2}>\tilde{\Delta} / \tilde{a}$, and the critical transverse wave vectors $\vec{q}_{c}$ are those that lie on a circle centered at $\quad \vec{q}_{0}=\left(0, q_{0, y}\right)$ with radius $R=\left|\vec{q}_{c}-\vec{q}_{0}\right|$ $=\sqrt{q_{0, y}^{2}-\tilde{\Delta} / \tilde{a}}$. Otherwise, for $q_{0, y}^{2}<\tilde{\Delta} / \tilde{a}$ the most unstable mode is $\vec{q}_{c}=\left(0,-q_{0, y}\right)$ and the convective threshold takes the form $\left|F_{c}\right|^{2}=1+\left\{\left[\tilde{\Delta}-\gamma_{2} \rho /(4 \tilde{a})\right] /\left(\gamma_{1}+\gamma_{2}\right)\right\}^{2}$. In both cases, the frequency of oscillations of the unstable modes at the convective threshold of instability is given by [13]

$$
\omega(\vec{q})=\operatorname{Im} \lambda_{+}\left(\vec{q}, F_{c}\right)=\frac{\gamma_{1} \gamma_{2}\left[\Delta_{2}-\Delta_{1}+q^{2}\left(a_{2}-a_{1}\right)+\rho q_{y}\right]}{\gamma_{1}+\gamma_{2}} .
$$

Hereafter we only consider the first case $\left(q_{0, y}^{2}-\tilde{\Delta} / \tilde{a}>0\right)$ for which pattern formation is expected [13].

The second threshold, $F_{a}\left(F_{a}>F_{c}\right)$ corresponds to the absolute instability. No analytical expression for $F_{a}$ is known, but can be calculated through the numerical solution of a saddle point problem $[13,30,31]$. At the absolute threshold the most unstable modes are still TW's, whose wave vector and frequency of oscillation are determined through the same formulas found for the convectively unstable regime, such as Eq. (6).

\section{Signal-idler synchronization}

In the absolutely unstable regime $\left(F>F_{c}\right)$ any initial random perturbation of the steady state takes the system to a final state that consists of an exact TW solution [see Eq. (14)]. In particular, due to symmetry breaking caused by the walk off $\rho \neq 0$, the selected wave vectors $\vec{q}=\left(q_{x}, q_{y}\right)$ always satisfies $q_{x}=0$ [23]. These nonlinear modes are not intensity patterns but rather phase patterns, one for the signal and one for the idler. The result is that, because of the deterministic nature of the instability in this regime, the correlation between signal and idler in the selected pattern is complete.

In the convectively unstable regime $\left(F_{c}<|F|<F_{a}\right)$ only NSS's exist [30,33]. In this regime perturbations are unstable but their advection speed is larger than their spreading velocity and thus new structures are continuously formed by spontaneous amplification of noise. In fact, the patterns are not stationary, but drift in the $y$ direction with the same advection velocity. NSS's are stochastic, as shown in a detailed analysis in Refs. [30,34], and their selection is dynamical, i.e., there is a continuous excitation of all the unstable modes both in the signal and the idler fields. Although many TW solutions are excited and grow, some modes, those closer to the condition $q_{x}=0$, are particularly favored. This stems from the symmetry breaking convective term, which makes 

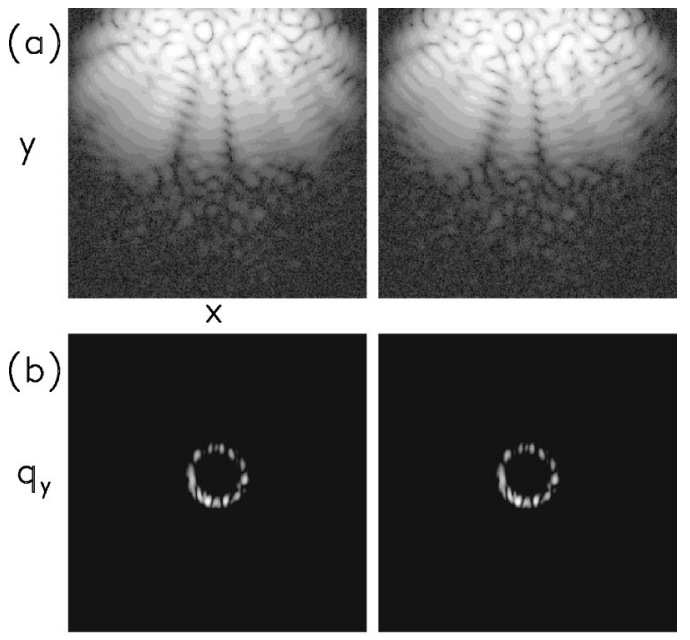

$q_{x}$

FIG. 1. Signal ( $A_{1}$, left column) and idler $\left(A_{2}\right.$, right column $)$ transverse fields in the convectively unstable regime at time $t$ =475. (a) Field intensities $\left|A_{1}\right|$ and $\left|A_{2}\right|$. (b) Fourier transforms $\left|A_{1, q}\right|$ and $\left|A_{2, q}\right|$ (far field). Parameters are $\gamma_{0}=1, \gamma_{1}=0.985, \gamma_{2}$ $=1.015, \quad a_{0}=0.125, \quad a_{1}=0.25375, \quad a_{2}=0.24625, \quad \Delta_{0}=0, \quad \Delta_{1,2}$ $=-0.2, \rho=0.25, \epsilon_{0}=\epsilon_{1,2}=9 \times 10^{-14}$, and $E_{0}=1.005$. Signal and idler transverse patterns, which drift in the $y$ direction, show essentially the same macroscopic noise-sustained structure in intensity. The integration window had a total size of $160 \times 160$ normalized spatial units, while only a central region of $80 \times 80$ units is shown here. The spatial width of the super Gaussian pump beam is 56 units.

the spreading velocity of perturbations with $q_{x}=0$ the largest in the system [30].

In the fully nonlinear stage of the convectively unstable regime, excited modes of opposite wave vector can reach a macroscopic size and interfere, generating intensity stripes [13]. These peculiarities of NSS are clearly observable in Fig. 1, which shows a snapshot of (a) near and (b) far field of the signal and idler in the convective regime. From the figure it is also noticeable that a large correlation between signal and idler exists in this regime. Let us stress that this behavior is not trivial, because the NSS's in the two fields are generated by the spontaneous amplification of two independent stochastic processes. To give evidence of the existence of a strong dynamical correlation-synchronization-in the nonlinear convective regime, the amplitude of the real and imaginary parts of the signal and idler fields are reproduced in Figs. 2 and 3.

\section{DYNAMICAL CORRELATION IN THE LINEAR REGIME}

In order to understand the synchronization phenomena between the signal and the idler fields, for which we have given numerical evidence above, we consider the dynamics in the linearized regime, i.e., for small field amplitudes around the trivial solution $A_{1,2}=0, A_{0}=E_{0} /\left(1+i \Delta_{0}\right)$. To avoid cumbersome expressions, analytical results are derived in this section for the particular case $K_{0}=1, \Delta_{0}=0, \gamma_{1,2}=\gamma$, and
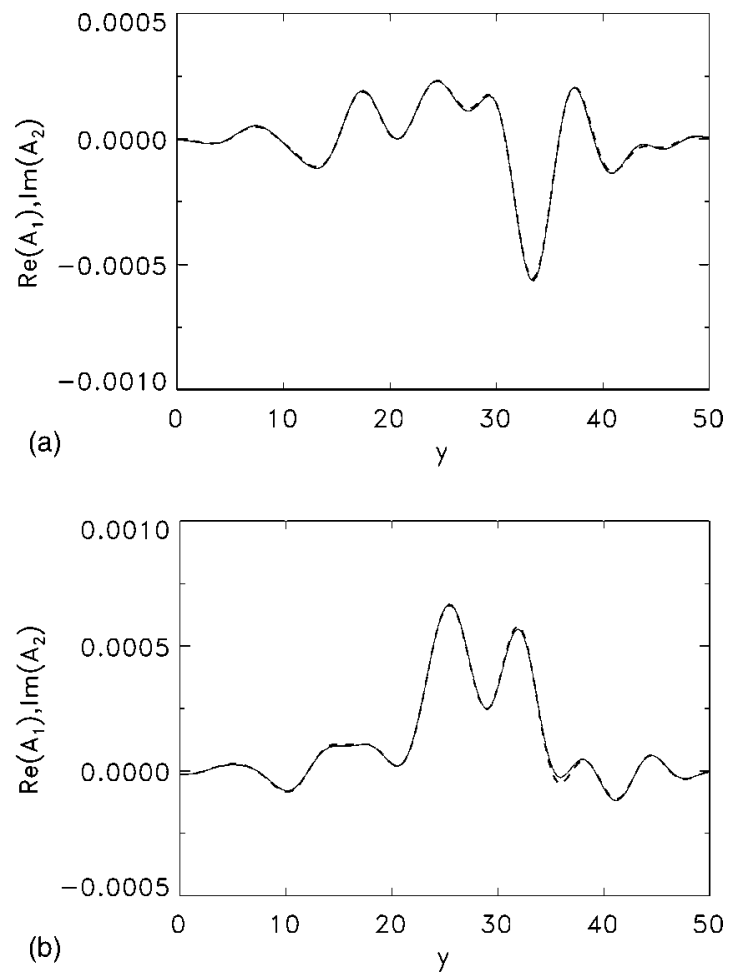

FIG. 2. (a) $\operatorname{Re}\left(A_{1}\right)$ (solid curve) and $\operatorname{Im}\left(A_{2}\right)$ (dashed curve) as a function of the space variable $y$, corresponding to the patterns of Fig. 1 at $x=0$, which is in the region of well-developed structures. (b) The same for $\operatorname{Re}\left(A_{2}\right)$ and $\operatorname{Im}\left(A_{1}\right)$.

$\epsilon_{1,2}=\epsilon$. We introduce the far-field components (Fourier transform)

$$
A_{i}(\vec{r}, t)=\frac{1}{2 \pi} \sum_{\vec{q}} A_{i, \vec{q}}(t) \exp (i \vec{q} \cdot \vec{r})
$$

with $i=1,2$. By linearizing Eqs. (2) and (3) around the off state the following equations are obtained for the Fourier modes:

$$
\begin{aligned}
\partial_{t} A_{1, \vec{q}}= & \gamma\left[-\left(1+i \Delta_{1}\right) A_{1, \vec{q}}-i a q^{2} A_{1, \vec{q}}+F A_{2,-\vec{q}}^{*}\right] \\
& +\sqrt{\epsilon} \psi_{1}(\vec{q}, t)
\end{aligned}
$$

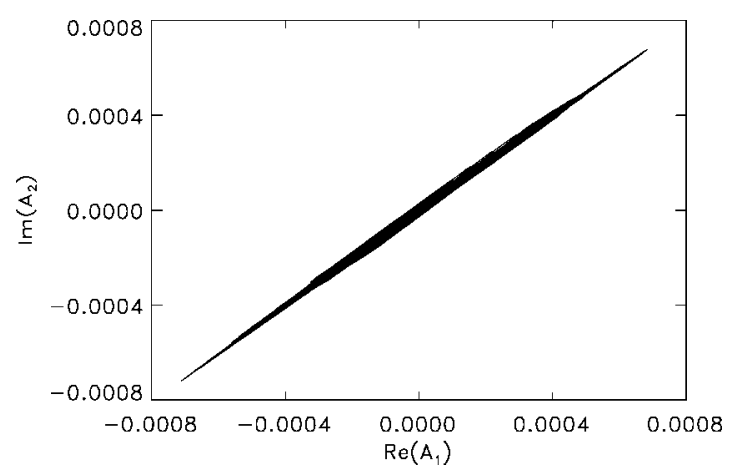

FIG. 3. Signal-idler synchronization can be observed by considering the time evolution in the $\left[\operatorname{Re}\left(A_{1}\right), \operatorname{Im}\left(A_{2}\right)\right]$ phase plane for the same integration of the previous figure. A similar picture is obtained in the $\left[\operatorname{Re}\left(A_{2}\right), \operatorname{Im}\left(A_{1}\right)\right]$ plane. 


$$
\begin{aligned}
\partial_{t} A_{2,-\vec{q}}^{*}= & \gamma\left[-\left(1-i \Delta_{2}\right) A_{2,-\vec{q}}^{*}+i a q^{2} A_{2,-\vec{q}}^{*}+i \rho q_{y} A_{2,-\vec{q}}^{*}\right. \\
& \left.+F^{*} A_{1, \vec{q}}\right]+\sqrt{\epsilon} \psi_{2}^{*}(-\vec{q}, t),
\end{aligned}
$$

where $\psi_{1,2}(\vec{q}, t)$ are Gaussian white noises in the Fourier space, defined through the expansion $\xi_{1,2}(\vec{r}, t)$ $=1 / 2 \pi \sum_{\vec{q}} \psi_{1,2}(\vec{q}, t) \exp (\overrightarrow{i q} \cdot \vec{r}), \quad$ with $\quad$ correlation: $\left\langle\psi_{i}(\vec{q}, t) \psi_{j}^{*}\left(\vec{q}^{\prime}, t^{\prime}\right)\right\rangle=2 \delta\left(\vec{q}-\vec{q}^{\prime}\right) \delta\left(t-t^{\prime}\right) \delta_{i j}$.

The correlation between the fields can be explained in terms of the eigenvectors $\vec{\Lambda}_{ \pm}(\vec{q})$ for $\left[A_{1, q_{c}}, A_{2,-}^{*} \vec{q}_{c}\right]^{T}$ associated with the eigenvalues $\lambda_{ \pm}$introduced in Eq. (5). In the critical case they can be written as

$$
\begin{gathered}
\vec{\Lambda}_{+}\left(\vec{q}_{c}\right)=\left[1,-i \exp \left(-i \phi_{0}\right)\right]^{T} \exp \left(i \vec{q}_{c} \cdot \vec{r}\right), \\
\vec{\Lambda}_{-}\left(\vec{q}_{c}\right)=\left[1, i \exp \left(-i \phi_{0}\right)\right]^{T} \exp \left(i \vec{q}_{c} \cdot \vec{r}\right),
\end{gathered}
$$

where $\phi_{0}$ is the phase of $A_{0}$. At threshold, $|F|=1$ and $\vec{\Lambda}_{+}\left(\vec{q}_{c}\right)$ defines the direction in the functional space along which the instability takes place. The form of eigenvectors (9) agrees with our numerical findings of synchronization. The unstable eigenvector direction defines a relationship between the signal mode and idler critical modes in such a way that $A_{1, \vec{q}_{c}}=i \exp \left(i \phi_{0}\right) A_{2,-\vec{q}_{c}}^{*}$. This relation between critical modes is also present below threshold and explains the observed signal-idler correlation. More precisely, decomposing the Fourier amplitudes in terms of the critical eigenvectors, we have

$$
\left[A_{1, \vec{q}_{c}}, A_{2,-\vec{q}_{c}}^{*}\right]^{T}=\alpha_{\vec{q}_{c}} \vec{\Lambda}_{+}\left(\vec{q}_{c}\right)+\beta_{q_{c}} \vec{\Lambda}_{-}\left(\vec{q}_{c}\right) .
$$

This expansion implies that

$$
\begin{aligned}
& \alpha_{\vec{q}_{c}}=A_{1, \vec{q}_{c}}+i A_{2,-\vec{q}_{c}}^{*} e^{i \phi_{0},}, \\
& \beta_{\vec{q}_{c}}=A_{1, \vec{q}_{c}}-i A_{2,-\vec{q}_{c}}^{*} e^{i \phi_{0}},
\end{aligned}
$$

and the following independent equations are derived for the modes that actually drive the dynamics in the convective regime:

$$
\begin{gathered}
\dot{\alpha}_{\vec{q}_{c}}=\gamma\left[|F|-1+i \omega\left(\vec{q}_{c}\right)\right] \alpha_{\vec{q}_{c}}+\psi_{\alpha}\left(\vec{q}_{c}, t\right), \\
\dot{\beta}_{\vec{q}_{c}}=-\gamma\left[|F|+1-i \omega\left(\vec{q}_{c}\right)\right] \beta_{\vec{q}_{c}}+\psi_{\beta}\left(\vec{q}_{c}, t\right),
\end{gathered}
$$

where $\omega(\vec{q})$ is given by Eq. (6) and we have introduced independent noise processes $\psi_{\alpha, \beta}=\psi_{1}(\vec{q}, t)$ $\pm i \psi_{2}^{*}(-\vec{q}, t) e^{i \phi_{0}}$.

From these equations it is clear that all modes indicated by $\beta_{q}$ are damped for any value of pumping $|F|$, while all modes defined as $\alpha_{q}$ are damped only below threshold, i.e., for $|F|<1$. This means that the destabilization of the trivial solution takes place along the manifold for which $\beta_{q_{c}}$ modes are damped. The consequence of the fact that $\beta_{q_{c}}(t) \rightarrow 0$ asymptotically, even in the convective regime, is the growth of a huge correlation between signal and idler spatial components in the far field. In fact, definition (11) and the result just found imply $A_{1, \vec{q}_{c}} \sim i A_{2,-\vec{q}_{c}}^{*} e^{i \phi_{0}}$. This correlation induces the corresponding correlation in the near field. For a resonant pump field $\left(\Delta_{0}=\phi_{0}=0\right)$, we have

$$
\begin{aligned}
A_{1}(\vec{r}, t) & =\frac{1}{2 \pi} \sum_{\vec{q}} A_{1, \vec{q}}(t) \exp (i \vec{q} \cdot \vec{r}) \\
& \approx \frac{1}{2 \pi} \sum_{\vec{q}} i A_{2,-\vec{q}}^{*}(t) \exp (i \vec{q} \cdot \vec{r})=i A_{2}^{*}(\vec{r}, t),
\end{aligned}
$$

at least if the sums are restricted to the Fourier modes on the ring of most unstable modes. This analysis is strictly valid only in the linearized regime, i.e., for small field amplitudes; nonetheless, the nonlinear convective regime preserves such property, as shown by numerical simulations of the full nonlinear equations (1)-(3) and the nonlinear solutions discussed in the following section.

\section{NONLINEAR SOLUTIONS}

A family of nonlinear, exact solutions of Eqs. (1)-(3), can be found analytically. In the limit of $\rho=0$, these solutions become the TW solutions found by Longhi in Ref. [36] for a NDOPO. These solutions are

$$
\begin{aligned}
{\left[A_{0}, A_{1}, A_{2}\right]^{T}=} & {\left[\bar{A}_{0}, \bar{A}_{1} \exp (i \vec{q} \cdot \vec{r}+i \omega t+i \Phi),\right.} \\
& \left.\bar{A}_{2} \exp (-i \vec{q} \cdot \vec{r}-i \omega t-i \Phi)\right]^{T},
\end{aligned}
$$

where

$$
\begin{gathered}
\bar{A}_{0}=\frac{E_{0}+i C \exp (i \Psi)}{1+i \Delta_{0}}, \\
\bar{A}_{1}=\bar{A}_{2}=\sqrt{C / 2 K_{0}} \exp (i \Psi / 2),
\end{gathered}
$$

and the dispersion relation $\omega(\vec{q})$ is given by Eq. (6). The intensity of the TW signal and idler solutions is given by

$$
C /\left(2 K_{0}\right)=\frac{-\left(1-\Delta_{0} \Delta_{e}\right) \pm \sqrt{K_{0}^{2}\left|E_{0}\right|^{2}-\left(1+\Delta_{0} \Delta_{e}\right)^{2}}}{K_{0}}
$$

where $\left(\gamma_{1}+\gamma_{2}\right) \Delta_{e}=\tilde{\Delta}+q^{2} \tilde{a}+\gamma_{2} q_{y} \rho$. The global signalidler phase $\Psi$ is

$$
2|F| \cos (\Psi)=\Delta_{e}+\frac{\gamma_{1}-\gamma_{2}}{\gamma_{1} \gamma_{2}} \omega(\vec{q}),
$$

while the relative signal-idler phase $\Phi$ is arbitrary, as follows from symmetry considerations [24]. The domain of existence of these TW solutions in the plane $\left(q, E_{0}\right)$ is determined by the condition $C>0$; thus, for $1-\Delta_{0} \widetilde{\Delta}>0$ only the positive sign in Eq. (16) is acceptable, and TW's exist for $|F|>F_{c}$ $=1$. At threshold $|F|=F_{c}$ and the transverse wave vectors of the TW's lie on the circle centered at $\vec{q}_{0}$ with radius $R$ (i.e., 


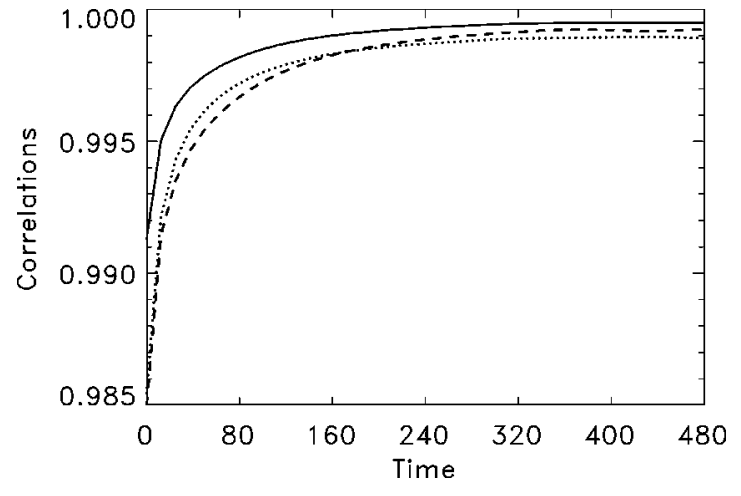

FIG. 4. Signal-idler correlations as a function of time for a characteristic realization of Eqs. (1)-(3) in the convective regime. The solid, dotted, and dashed curves represent, respectively, $\Gamma\left[\left|A_{1}\right|,\left|A_{2}\right|\right], \Gamma\left[\operatorname{Re}\left(A_{1}\right), \operatorname{Im}\left(A_{2}\right)\right]$ and $\Gamma\left[\operatorname{Re}\left(A_{2}\right), \operatorname{Im}\left(A_{1}\right)\right]$. Parameters are the same as of Fig. 1.

$\left.\Delta_{e}=0\right)$. Note that only in the case $\gamma_{1}=\gamma_{2}$ the global phase is invariant at $|F|=F_{c}$, and any pair of signal-idler TW has the same global phase.

It is important to note that the most unstable modes of the linear stability analysis, i.e., those identified by the wave vector $\vec{q}_{c}$ and the relative frequency [Eq. (6)], belong to the family of exact nonlinear TW solutions. In particular, by substituting the expression of $\vec{q}_{c}$ into the formula for $\Delta_{e}$, all the most unstable modes (those on the ring) satisfy $\Delta_{e}=0$. These TW's satisfy for resonant pump field $\left(\Delta_{0}=0\right)$ the relation $A_{1}=i A_{2}^{*}$. This relation is not a symmetry of Eqs. (1)-(3), but it follows the direction of instability. This condition means that a complete correlation exists between the real and imaginary parts of signal and idler.

\section{TIME CORRELATION IN THE CONVECTIVE REGIME}

To better quantify the phenomenon of synchronization of the two stochastic fields $A_{1}$ and $A_{2}^{*}$ in the convective regime, the following time correlation function $\Gamma$ is introduced:

$$
\begin{aligned}
\Gamma[P(x, y, t), Q(x, y, t)]= & \Gamma(t)=\iint[(P-\langle P\rangle)(Q \\
& -\langle Q\rangle)] d x d y /[\sqrt{\sigma(P)} \sqrt{\sigma(Q)}],
\end{aligned}
$$

where $P, Q$ can be any of the following quantities: $\operatorname{Re}\left(A_{1}\right), \operatorname{Re}\left(A_{2}\right), \operatorname{Im}\left(A_{1}\right), \operatorname{Im}\left(A_{2}\right),\left|A_{1}\right|,\left|A_{2}\right|$, determined at a certain point $(x, y)$ and time $t,\langle\rangle$ indicates their mean value in the transverse plane and $\sigma()$ is their corresponding variance. In Fig. 4 it can be observed that certain correlations calculated for the NSS's of Fig. 1 asymptotically reach the unity as the NSS's develop. In practice, the uncorrelated initial condition is substituted, as time evolves, by highly correlated NSS's in the two components. Then, after a characteristic transition time, which depends on the system size and the walk-off coefficient, the signal and idler evolutions become synchronized stochastic processes. This synchronization is not only present in integrated quantities as $\Gamma(t)$ but also present point to point, as shown in Figs. 2 and 3. This means that the phenomenon is spatially distributed. In practice, the nonlinear pattern is constituted by the sum of all convectively unstable Fourier modes, i.e., nonlinear exact solutions, which can be excited at the same time in this regime.

The degree of correlation between the fields $A_{1}$ and $A_{2}^{*}$ depends on the difference of cavity decay rates and on the noise intensity. The correlation is originated from an interplay between noise and nonlinear dynamics. Directions perpendicular to the unstable manifolds are damped, as shown in Eq. (12). One has to to compare the damping ratio $\gamma(1$ $+|F|$ ) with the noise strength $\epsilon$. For larger noise intensity or lower damping rates, the correlation is decreased. If $\gamma_{1}$ $\neq \gamma_{2}$ the global phase is not the same for the $q$ modes in the ring of the far field [see Eq. (17)] and the correlation is also degraded. However, even in this case the signal-idler near field synchronization is due to the superposition of nonlinear traveling waves with global signal-idler phase fixed, being the dominant contribution from the far field the modes with $q_{x}=0$.

\section{CONCLUSIONS}

In conclusion, a theoretical analysis of synchronization in noise-sustained patterns for intracavity parametric down conversion has been presented. This phenomenon occurs in the convectively unstable regime, where noise-sustained structures can be generated by spontaneous amplification of noise. For pump values between the convective and absolute threshold of a pattern forming instability, the destabilization of the steady state leads to the formation of stochastic, selforganized structures in the down converted signal and idler fields. In the linearized regime, the most unstable modes are traveling waves that satisfy the condition $A_{1} \simeq i A_{2}^{*}$; such correlation is also a characteristic of a particular family of nonlinear solutions. Hence, the result of the destabilization is the excitation of convectively unstable nonlinear modes that present the same correlation $\left(A_{1} \simeq i A_{2}^{*}\right)$ at each spatial point and for all times. The final result is that signal and idler are synchronized stochastic spatiotemporal fields.

Our analysis provides a remarkable example of synchronization of macroscopic noise-sustained structures with a vectorial degree of freedom.

\section{ACKNOWLEDGMENTS}

G.I. acknowledges helpful discussions with P. Scotto. We acknowledge financial support from MCyT (Spain) Project Nos. PB97-0141-C02-02, BFM2000-1108, BFM2001-0341C02-02, Spanish-Italian Acción Integrada HI2000-0027, and CSIC (Spain)-CONICET (Argentine) collaboration program. 
[1] C. Huygens, J. Scavants XI, 79 (1665); XII, 86 (1665). For a recent review of synchronization processes see S. Strogatz, Sync: The Emerging Science of Spontaneous Order (Hyperion, New York, 2003).

[2] A.T. Winfree, The Geometry of Biological Time (Springer, New York, 1980).

[3] A.T. Winfree, J. Theor. Biol. 16, 15 (1967).

[4] Y. Kuramoto, in Proceedings of the International Symposium on Mathematical Problems in Theoretical Physics, edited by H. Araki, Lecture Notes in Physics Vol. 39 (Springer, Berlin, 1975); Chemical Oscillations, Waves, and Turbulence (Springer, Berlin, 1984).

[5] L.M. Pecora and T.L. Carroll, Phys. Rev. Lett. 64, 821 (1990).

[6] M.G. Rosenblum, A.S. Pikovsky, and J. Kurths, Phys. Rev. Lett. 76, 1804 (1996).

[7] N.F. Rulkov, M.M. Sushchik, L.S. Tsimring, and H.D.I. Abarbanel, Phys. Rev. E 51, 980 (1995).

[8] L. Kocarev and U. Parlitz, Phys. Rev. Lett. 76, 1816 (1996).

[9] C. Serrat, M. Torrent, J.G. Ojalvo, and R. Vilaseca, Phys. Rev. A 64, 041802(R) (2001).

[10] R. Toral, C. Mirasso, E. Hernández-García, and O. Piro, Chaos 11, 665 (2001).

[11] A. Amengual, E. Hernández-García, R. Montagne, and M. San Miguel, Phys. Rev. Lett. 78, 4379 (1997).

[12] E. Hernandez-García, M. Hoyuelos, P. Colet, R. Montagne, and M. San Miguel, Int. J. Bifurcation Chaos Appl. Sci. Eng. 9, 2257 (1999); E. Hernandez-García, M. Hoyuelos, P. Colet, and M. San Miguel, Phys. Rev. Lett. 85, 744 (2000).

[13] G. Izús, M. Santagiustina, M. San Miguel, and P. Colet, J. Opt. Soc. Am. B 16, 1592 (1999).

[14] F.T. Arechi, S. Boccaletti, and P.L. Ramazza, Phys. Rep. 318, 1 (1999); see also special issue of J. Opt. B: Quantum Semiclassical Opt. 1, 1 (1999).

[15] G.-L. Oppo, M. Brambilla, and L.A. Lugiato, Phys. Rev. A 49, 2028 (1994).

[16] G.J. de Valcarcel, K. Staliunas, E. Roldan, and V.J. SanchezMorcillo, Phys. Rev. A 54, 1609 (1996).

[17] S. Longhi, Phys. Scr. 56, 611 (1997).

[18] K. Staliunas and V. Sánchez-Morcillo, Phys. Rev. A 57, 1454 (1998).
[19] G.L. Oppo, A.J. Scroggie, and W.J. Firth, J. Opt. B: Quantum Semiclassical Opt. 1, 133 (1999).

[20] M. Le Berre, D. Leduc, E. Ressayre, and A. Tallet, J. Opt. B: Quantum Semiclassical Opt. 1, 153 (1999); M. Tlidi, M. Le Berre, E. Ressayre, A. Tallet, and L. Di Menza, Phys. Rev. A 61, 043806 (2000).

[21] G.-L. Oppo, A.J. Scroggie, and W.J. Firth, Phys. Rev. E 63, 066209 (2001).

[22] S. Trillo, M. Haelterman, and A. Sheppard, Opt. Lett. 22, 970 (1997); N. Kutz, T. Ernaux, S. Trillo, and M. Haelterman, J. Opt. Soc. Am. B 16, 1936 (1999).

[23] M. Santagiustina, P. Colet, M. San Miguel, and D. Walgraef, Opt. Lett. 23, 1167 (1998).

[24] G. Izús, M. Santagiustina, and M. San Miguel, Opt. Lett. 25, 1454 (2000); Phys. Rev. E 64, 056231 (2001).

[25] G. Izús, M. San Miguel, and D. Walgraef, Phys. Rev. E 66, 036228 (2002).

[26] M. Santagiustina, E. Hernadez-García, M. San Miguel, A.J. Scroggie, and G.-L. Oppo, Phys. Rev. E 65, 036610 (2002).

[27] D. Gomila, P. Colet, M. San Miguel, and G.-L. Oppo, IEEE J. Quantum Electron. 39, 238 (2003).

[28] M. Vaupel, A. Matre, and C. Fabre, Phys. Rev. Lett. 83, 5278 (1999).

[29] S. Ducci, N. Treps, A. Maitre, and C. Fabre, Phys. Rev. A 64, 023803 (2001).

[30] M. Santagiustina, P. Colet, M. San Miguel, and D. Walgraef, Phys. Rev. E 58, 3843 (1998).

[31] H. Ward, M.N. Ouarzazi, M. Taki, and P. Glorieux, Eur. Phys. J. D 3, 275 (1998).

[32] M. Taki, N. Ouarzazi, H. Ward, and P. Glorieux, J. Opt. Soc. Am. B 17, 997 (2000).

[33] M. Santagiustina, P. Colet, M. San Miguel, and D. Walgraef, Phys. Rev. Lett. 79, 3633 (1997).

[34] M. Santagiustina, P. Colet, M. San Miguel, and D. Walgraef, Opt. Express 3, 63 (1998).

[35] K. Babcock, G. Ahlers, and D. Cannell, Phys. Rev. Lett. 67, 3388 (1991); M. Scherer and G. Ahlers, Phys. Rev. E 65, 051101 (2002).

[36] S. Longhi, Phys. Rev. A 53, 4488 (1996). 